\title{
Checking Properties of Heap-Manipulating Procedures with a Constraint Solver
}

\author{
Mandana Vaziri and Daniel Jackson \\ Laboratory for Computer Science \\ Massachusetts Institute of Technology \\ Cambridge, Massachusetts, USA \\ $\{$ vaziri,dnj\}@lcs.mit.edu
}

\begin{abstract}
A method for finding bugs in object-oriented code is presented. It is capable of checking complex user-defined structural properties - that is, of the configuration of objects on the heap - and generates counterexample traces with no false alarms. It requires no annotation beyond the specification to be checked, and is fully automatic.

The method relies on a three-step translation: from code to a formula in a first-order relational logic, then to a propositional formula, and finally to conjunctive normal form. An off-the-shelf SAT solver is then used to find a solution that constitutes a counterexample.

This underlying scheme, presented previously, does not scale readily. In this paper, we show how a suite of optimizations results in much improved scalability. The optimizations are based on a special treatment of relations that are known to be functional, and target all steps. The effect of the optimizations is demonstrated by application to the analysis of a red-black tree implementation.
\end{abstract}

\section{Introduction}

In previous work [13, we developed a scheme for finding bugs in Java code using a SAT solver. The code, along with a declarative specification, is translated first into a first-order relational formula, and from there into a propositional formula whose satisfying assignments correspond to counterexamples - traces that violate the specification.

This approach is inherently intractable, so it is essential to eliminate any unnecessary causes of complexity. One such cause that we have been aware of since the start is an uneconomical representation of fields. A field of a class is mathematically a function, since it maps an object to at most one other object, and its possible values can be encoded with a bit string of length $\log (n)$ for each domain value, corresponding to the integer index of the field's value, drawn from $n$ possible values. But our translation scheme, which was designed for a more general modelling language [11], encodes a function as a relation, with $n^{2}$ bits, one for each combination of domain and range values.

Unfortunately, it is not obvious how to exploit the tighter encoding of functions, since it is not well suited to applying the relational operators, especially 
the image operator, which appears frequently, due to the translation of set and get operations on fields. Introducing an additional representation of each function in the standard form worsens performance, since the tighter encoding is now an addition, rather than a replacement.

This paper shows how to make effective use of the tighter encoding of functions. It exploits the fact that most of the relational formulas arising from an object-oriented program take a stylized form. A transformation of this form into another relational formula with additional relational variables is given, which can be translated into CNF, using some specialized logical simplifications, without any additional boolean variables.

Section 2 gives an overview of the basic approach, which we have described previously [13]. Section 3 explains the generic scheme for translating relational formulas into propositional formulas on which our previous work relied 11]. The new content of the paper is presented in Section 4, and evaluated experimentally in Section 5. The paper closes with related work (Section 6) and conclusions (Section 7).

\section{Encoding Object-Oriented Code in Alloy}

\section{$2.1 \quad$ Overview and Illustration}

Our analysis 13 gives a bounded interpretation to a fragment of Java code, by unwinding loops some number of times and by limiting the number of heap cells of each type. Procedure calls are handled by inlining; there is currently no treatment of recursion. The Java code is translated into Alloy [12], a modelling language based on first-order logic. User-defined properties are also expressed in Alloy, which allows succinct declarative expressions of complex structural properties. For example, the fact that a list is acyclic can be expressed using the transitive closure operator, and that red-black trees have the same number of black nodes on each path is expressible with set cardinalities. Given an Alloy model for the program, the Alloy Analyzer [11] is then used, together with a user-provided bound on the number of heap cells, to check properties, and to find counterexamples when they do not hold.

Our method differs from other verification approaches in that it targets properties of the heap. It considers all the possible initial configurations within finite bounds. If there is a property violation, it will determine the initial configuration responsible for it, as part of the counterexample trace. The analysis typically accounts for billions of cases, which would not be feasible with testing alone. The SAT solver - the Alloy Analyzer's core engine - works in a goal-oriented fashion. Since it tries to satisfy a boolean formula, it does not go through all the executions in turn, and may effectively search multiple executions at once. Our approach also does not perform approximations beyond considering a finite instance of the code. This means that there are no false error reports.

Consider the swapTail procedure (Figure 1), which purportedly takes two linked lists and swaps their tails. We use our analysis to check whether the 


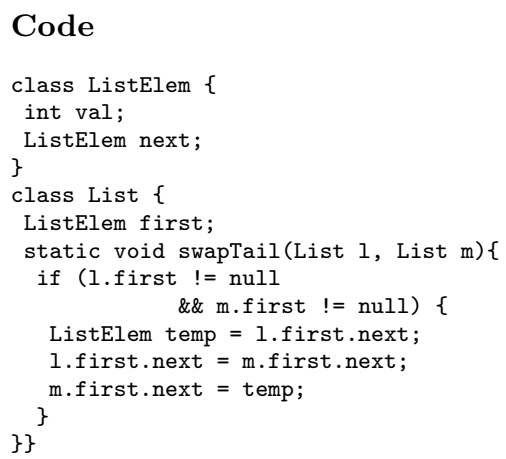

\section{Code}

class ListElem \{

int val;

ListElem next;

\}

class List \{

ListElem first;

static void swapTail(List 1 , List $m$ ) \{

if (l.first != null

\&\& m.first $!=$ null) \{

ListElem temp $=1$.first.next;

1.first.next $=\mathrm{m}$.first.next;

m.first.next $=$ temp;

\}

\}\}

\section{Computation Graph}

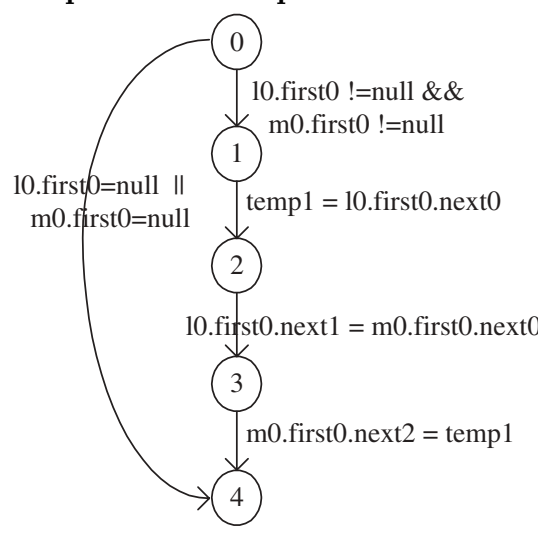

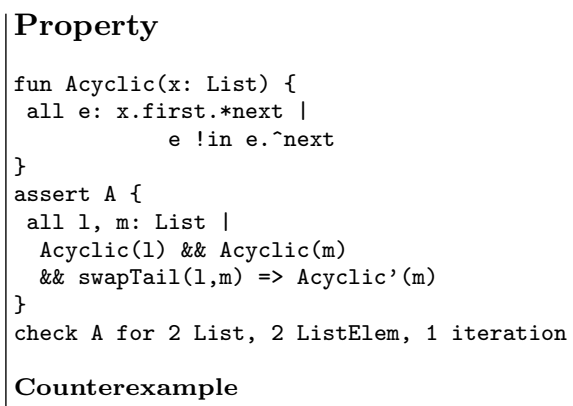
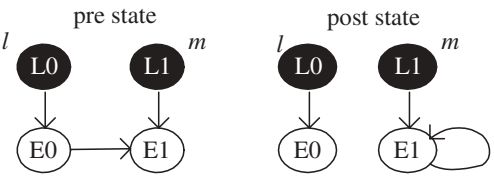

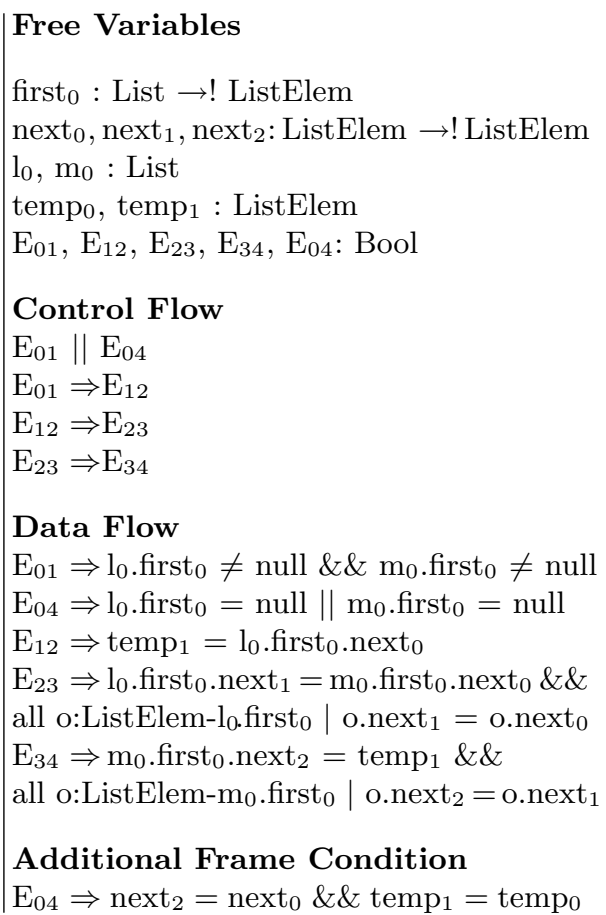

Fig. 1. An Example of a Procedure and its Analysis

swapTail procedure preserves the property that its inputs are acyclic. We write the Alloy specification shown in Figure 11 The assertion A states that: for all Lists 1 and $\mathrm{m}$, if they are both acyclic in the initial state, and swapTail $(1, \mathrm{~m})$ is called, then $\mathrm{m}$ is acyclic in the post state, which is indicated by the prime sign on the last appearance of the function Acyclic. 
The auxiliary function Acyclic( $\mathrm{x}$ : List) defines the constraint that a list is acyclic, by stating that for all ListElems e reachable from $\mathrm{x}$.first, where $\mathrm{x} . \mathrm{first}$ is included, e is not reachable from itself.

The command that follows (check A) instructs the tool to use 2 heap cells per type and 1 iteration. The analysis produces a counterexample shown partially in Figure 1. Black circles represent heap cells of type List, and white ones are of type ListElem. Arrows represent fields. In the pre-state, list $m$ is a list of one element, and 1 of two, and they share an element. In the post-state, $\mathrm{m}$ is a list with an element whose next field points to itself, and is therefore cyclic, violating the assertion.

\subsection{Extracting a Computation Graph}

We translate a Java procedure into an Alloy formula whose models correspond to executions. It is composed of two subformulas, encoding the control and data flow of the procedure.

We start with the procedure's control flow graph (CFG), with nodes representing its control points, and edges labeled with either Java statements or control predicates. We unroll the loops in the CFG up to a number of iterations (selected by the user), and inline procedure calls, to obtain a computation graph. For example, one unrolling of $a$; while $(p) s ; b$ gives the graph one would obtain as the standard CFG of $a$; if $(p) s$; assert ! $p$; b.

We rename variable and field names, in such a way that no path has two assignments to the same variable or sets the same field name, but parallel paths may share names (just as in single static assignment [3], but without $\phi$ functions). Renaming is done by providing an index for each variable at each node in the computation graph. In what follows we use name $(v, i)$ to denote the name of variable $v$ at node $i$.

Figure 1 shows the computation graph corresponding to the swapTail procedure. The edge between nodes (0) and (4) is traversed when the condition of the if statement is false.

\subsection{Encoding the State, Control, and Data Flow}

Variables in the Alloy formula are used to encode the state at the various control points of the procedure. We model a field of type $t$ appearing in class $c$ with a total function from $c$ to $t$. Each type has a special atom representing null. Local variables and formal parameters are modelled as scalars, which are represented by singleton sets in Alloy.

In addition, the variables of the Alloy formula include a boolean variable 1 $E_{i j}$ for each edge from node $i$ to $j$ in the computation graph. These are used to encode the control and data flow, and indicate whether an edge is traversed during an execution. The free variables for swapTail are shown in Figure [1] where a declaration of the form $f: A \rightarrow ! B$ says that $f$ is a relation from $A$ to

\footnotetext{
${ }^{1}$ Alloy does not have built-in booleans, but these can be easily encoded with sets.
} 
$B$, restrained to be a total function from $A$ to $B$, and $a: A$ declares $a$ to be a scalar of type $A$.

The control flow is encoded with a formula that captures when an edge is traversed. For each node $i$, let $i n(i)$ be the set of nodes having an outgoing edge to $i$, and out $(i)$ the set of nodes having an incoming edge from $i$. For each node $i$, we produce $\vee\left\{E_{j i} \mid j \in i n(i)\right\} \Rightarrow \vee\left\{E_{i k} \mid k \in\right.$ out $\left.(i)\right\}$, and the formula encoding the control flow is the conjunction of these formulas. These mean that if some node's incoming edge is traversed then some of its outgoing edges are also traversed. Infeasible paths are ruled out because some of the edges are labeled with control predicates, and these appear in the formula that encodes the data flow presented below. Note that if more than one outgoing edge is traversed, the constraint solver may generate an instance corresponding to more than one execution. But all these executions are feasible. It is a question of tool design which one is presented to the user. In the case of swapTail, the formula encoding control flow is shown in Figure 1.

We encode the data flow for each edge with a formula that indicates how variables are related before and after the execution of the statement corresponding to that edge. For each edge $e$ from node $i$ to $j$, we produce a formula: $E_{i j} \Rightarrow t$, where $t$ is the translation of the Java statement corresponding to $e$ into firstorder logic. The formula that encodes the data flow is then the conjunction of these formulas. This means that whenever $e$ is traversed, the effect of the Java statement encoded by $t$ is observed. The translation rules for Java statements are given in our earlier paper [13]. In the case of swapTail, the formula encoding data flow is shown in Figure 11.

In Alloy, the expression a.r, where $a$ is a set and $r$ is a relation, denotes the relational image of $a$ under $r$. So $l_{0}$. first $t_{0}$ denotes the image of $l_{0}$ under function first $_{0}$. The formula

$$
\text { all o: ListElem }-l_{0} \cdot \text { first }_{0} \mid \text { o.next } 1=0 . \text { next }_{0}
$$

is a frame condition. It means that for all ListElems other than $l_{0}$. first , the next relation remains the same. Alloy is a declarative language in which variables that are left unconstrained can take on any value. Frame conditions are then needed to say that certain variables remain the same when an update happens.

Finally, we conjoin a set of additional frame conditions. When an edge connects nodes $i$ and $j$ that assign a different index to a field or variable $v$, but $v$ is not modified by the statement associated with the edge, we produce the frame condition: $E_{i j} \Rightarrow \operatorname{name}(v, j)=\operatorname{name}(v, i)$. In the case of swapTail, the additional frame condition (shown in Figure 1) says that whenever $\mathrm{E}_{04}$ is traversed, the next relation and the temp variable remain the same.

\section{Translation to Propositional Logic}

In Alloy, every type consists of a set of atoms. The values of variables are relations, which are sets of tuples of atoms; and sets are treated as degenerate 
relations, consisting of a set of unary tuples. The user provides to our analysis a bound $n$ on the number of heap cells for each class, and this is used to set scopes for the Alloy Analyzer, i.e. the number of atoms of each type. The analyzer uses the scope to translate an Alloy formula to propositional logic. It allocates a matrix of $n^{2}$ boolean variables to each binary relation $r$ :

$$
\begin{gathered}
r_{11} \cdots r_{1 n} \\
\cdots \cdots \cdot \\
r_{n 1} \cdots r_{n n}
\end{gathered}
$$

where $r_{i j}$ is true if and only if $r$ maps atom $i$ of its domain to atom $j$ of its range. After having allocated boolean variables to all relations in the formula, the analyzer then proceeds to combine these matrices into matrices of boolean formulas. For example, given a set $a$, represented as a degenerate relation, the relational image $a . f$ of $a$ under relation $f$, gets the matrix:

$$
\begin{gathered}
\left(a_{1} \wedge f_{11}\right) \vee \cdots \vee\left(a_{n} \wedge f_{n 1}\right) \\
\cdots \\
\left(a_{1} \wedge f_{1 n}\right) \vee \\
\cdots \vee\left(a_{n} \wedge f_{n n}\right)
\end{gathered}
$$

which is the result of matrix multiplication, and states that atom $i$ is an element of $a . f$ if and only if there is some atom $j$ in $a$ such that $f$ maps $j$ to $i$. Other Alloy expressions are translated into matrices of boolean formulas in a similar way [11].

To translate formulas, the analyzer combines these matrices into a single propositional formula. For example, the formula $f=g$, where $f$ and $g$ are binary relations, becomes:

$$
\left(f_{11} \Leftrightarrow g_{11}\right) \wedge \cdots \wedge\left(f_{1 n} \Leftrightarrow g_{1 n}\right) \wedge \cdots \wedge\left(f_{n 1} \Leftrightarrow g_{n 1}\right) \wedge \cdots \wedge\left(f_{n n} \Leftrightarrow g_{n n}\right) .
$$

which states that variables $f$ and $g$ denote the same set of tuples: tuple $(i, j)$ is in $f$ if and only if it is in $g$.

Given a propositional formula, the analyzer then proceeds to transform it into conjunctive normal form (CNF) by renaming all subformulas with fresh propositional variables, and conjoining appropriate definitions for these variables to the whole formula [15]. This avoids the exponential blow-up in the size of the formula when it is translated into CNF using distributivity laws, but does increase the number of variables. An off-the-shelf SAT solver takes the CNF produced and attempts to find a model. In our case, a satisfying assignment corresponds to a counterexample, which is then output to the user in an appropriate fashion.

\section{Exploiting Properties of Functions}

A field declared in a class is modelled as a relation, but it always maps its object to exactly one other object (or to null). Mathematically, this is a function. By exploiting this fact, we can optimize different steps of the analysis presented in the previous section, with the goal of reducing the number of variables and 
clauses produced in the final CNF, since this will improve the SAT solver's performance.

The main optimization is a representation for functions that requires fewer boolean variables than the representation for general relations. However, this does not reduce the number of variables in the CNF, because the step that translates propositional formulas to CNF adds intermediate variables, and counteracts the benefit of the compact function representation. To obtain a real benefit we need two other kinds of optimizations: first a systematic introduction of variables in the first-order formula, and second a series of logical simplifications in the propositional formula. In the next sections, we describe how these optimizations work together to reduce the number of clauses and variables in the CNF.

\subsection{Function Representation}

A relation $f$ that is a total function maps each atom in its domain to exactly one atom in its range. By representing this atom as an integer in binary form, the encoding of $f$ requires only $\lfloor\log (n)\rfloor+1$ rather than $n$ boolean variables in each row. From this tighter encoding:

$$
\begin{aligned}
& f_{11} \cdots f_{1 l} \\
& \cdots \cdots \\
& f_{n 1} \cdots f_{n l}
\end{aligned}
$$

we can extract the standard, $n \times n$ representation:

$$
\begin{aligned}
& \begin{array}{cc}
\neg f_{11} \wedge \cdots \wedge \neg f_{1 l} & \neg f_{11} \wedge \cdots \wedge \neg f_{1(l-1)} \wedge f_{1 l} \\
\ldots & \ldots
\end{array} \\
& \neg f_{n 1} \wedge \cdots \wedge \neg f_{n l} \quad \neg f_{n 1} \wedge \cdots \wedge \neg f_{n(l-1)} \wedge f_{n l} \quad \ldots
\end{aligned}
$$

where the formula at row $i$ and column $j$ is true if and only if row $i$ in the compact representation of $f$ represents integer $j$. Note that since $\lfloor\log (n)\rfloor+1$ bits can represent more than $n$ values, we must add a side condition that constrains each row of the compact representation in such way that it represents an integer less than $n$.

If we incorporate this optimization in the Alloy Analyzer, this actually results in an increase in the number of variables in the final CNF. This is because the step that transforms propositional logic to CNF counteracts the gain of the compact representation, by renaming all the formulas in the converted matrix with propositional variables. So the resulting $\mathrm{CNF}$ has all the variables that it would have had without the compact representation, in addition to all the ones the representation introduces.

\subsection{Introducing Alloy Variables}

To avoid this problem, we first rename all subexpressions that are scalars, i.e. singleton sets, in the first-order formula. Most subformulas that appear in the translation of a fragment of Java code have the form: 


$$
v \cdot f_{1} \cdot \cdots \cdot f_{k_{1}}=u \cdot g_{1} \cdot \cdots \cdot g_{k_{2}},
$$

where $v$ and $u$ are scalars, and $f_{1}, \cdots, f_{k_{1}}$, and $g_{1}, \cdots, g_{k_{2}}$ are functions, encoding fields. We rename subexpressions of the form $a . f$ by introducing an Alloy variable $b$, and conjoin definitions of the form $b=a . f$ with the whole formula. Variable $b$ is a scalar since $a$ is a scalar and $f$ is a function. We obtain:

$$
\begin{gathered}
v_{1}=v \cdot f_{1} \wedge \cdots \wedge v_{k_{1}-1}=v_{k_{1}-2} \cdot f_{k_{1}-1} \wedge u_{1}=u . g_{1} \wedge \cdots \wedge u_{k_{2}}=u_{k_{2}-1} \cdot g_{k_{2}} \\
\wedge v_{k_{1}-1} \cdot f_{k_{1}}=u_{k_{2}} .
\end{gathered}
$$

The next section describes logical simplifications that allow a formula of the form $a . f=b$ to be translated compactly to CNF without adding any additional propositional variables. The CNF's for these formulas are then conjoined by taking the union of their clauses. Introducing an Alloy variable for the subexpression a. $f$ results in $\lfloor\log (n)\rfloor+1$ additional boolean variables. If this subexpression were translated to CNF without the introduction of Alloy variables and logical simplifications, it would result in at least $n^{2}$ additional boolean variables, since all the elements of the product (an $n \times n$ matrix) would be renamed.

\subsection{Logical Simplifications}

We now describe the logical simplifications that allow us to translate $a . f=b$ compactly to $\mathrm{CNF}$, without introducing any additional propositional variables. They take advantage of the fact that a scalar is represented by a collection of propositional formulas having the property that exactly one of them is true. Informally, the first two simplifications help because they push disjunctions down in the formula's syntax tree. Disjunctions are a source of blow-up when transforming to $\mathrm{CNF}$, and their effect is lessened if they are further away from the root.

Logical Simplification 1 Consider the formula:

$$
\left(A_{1} \wedge B_{1}\right) \vee \cdots \vee\left(A_{n} \wedge B_{n}\right)
$$

where $A_{i}$ and $B_{i}(1 \leq i \leq n)$ are boolean formulas. If exactly one of the $A$ formulas is true, then it can be easily seen that (1) is logically equivalent to:

$$
\left(\neg A_{1} \vee B_{1}\right) \wedge \cdots \wedge\left(\neg A_{n} \vee B_{n}\right)
$$

Logical Simplification 2 Consider the formula:

$$
\left(\left(A_{1} \wedge B_{1}\right) \vee \cdots \vee\left(A_{n} \wedge B_{n}\right)\right) \Leftrightarrow C
$$

where $A_{i}$ and $B_{i}(1 \leq i \leq n)$ are boolean formulas. If exactly one of the $A$ formulas is true, then it can be easily seen that (3) is logically equivalent to:

$$
\left(A_{1} \wedge\left(B_{1} \Leftrightarrow C\right)\right) \vee \cdots \vee\left(A_{n} \wedge\left(B_{n} \Leftrightarrow C\right)\right)
$$

Our final simplification is specific to the representation of integers, and relies on the fact that integers can be compared bit by bit. 
Definitions. A literal is either a propositional variable, or the negation of one. Given a literal $a$, let $\operatorname{var}(a)$ denote the propositional variable corresponding to $a$, and phase $(a)$ be $+(-)$ if $a$ is $\operatorname{var}(a)(\neg \operatorname{var}(a))$.

Logical Simplification 3. Let $A_{i}(1 \leq i \leq n)$ be a collection of formulas of the form $a_{1}^{i} \wedge \cdots \wedge a_{l}^{i}$, such that for all $i, j$, and for all $k(1 \leq k \leq l)$, $\operatorname{var}\left(a_{k}^{i}\right)=\operatorname{var}\left(a_{k}^{j}\right)$, and let $B_{i}$ be a similar collection. Consider the formula:

$$
A_{1} \Leftrightarrow B_{1} \wedge \cdots \wedge A_{n} \Leftrightarrow B_{n}
$$

If exactly one of the $A_{i}$ is true, and similarly for the $B_{i}$, and for all $i$ and $k$, phase $\left(a_{k}^{i}\right)=\operatorname{phase}\left(b_{k}^{i}\right)$, then it can be seen that (5) is logically equivalent to:

$$
\operatorname{var}\left(a_{1}^{1}\right) \Leftrightarrow \operatorname{var}\left(b_{1}^{1}\right) \wedge \cdots \wedge \operatorname{var}\left(a_{l}^{1}\right) \Leftrightarrow \operatorname{var}\left(b_{l}^{1}\right)
$$

\subsection{Using the Optimizations}

Let us now compute the number of clauses and variables obtained in the CNF for $v \cdot f_{1} \cdots \cdot f_{k_{1}}=u \cdot g_{1} \cdots \cdot g_{k_{2}}$ using our optimizations, to compare them to the case with no optimizations.

We have seen that $v \cdot f_{1} \cdots \cdot f_{k_{1}}=u \cdot g_{1} \cdots \cdot g_{k_{2}}$ can be transformed into a conjunction of $k_{1}+k_{2}$ formulas of the form $a . f=b$. Let $k$ denote $k_{1}+k_{2}$. Since conjunction of CNF can be obtained simply by taking union of clause sets, we can avoid variable introduction and obtain a formula of size $k \alpha$, if $a . f=b$ can be represented with $\alpha$ clauses.

In what follows, we compute $\alpha$, but first we introduce some notation. Consider translating the formula $a . f=b$ to CNF. The variable $a$ is a scalar and its compact representation requires $l$ boolean variables: $a_{1} \cdots a_{l}$, where $l$ denotes $\lfloor\log (n)\rfloor+1$, and $n$ is the scope. Function $f$ has a compact representation having $n l$ boolean variables: $f_{11} \cdots f_{1 l} \cdots f_{n 1} \cdots f_{n l}$.

Converting the compact representation of $a$ to the standard one results in a vector of $n$ elements. We use $A_{i}$ to denote the formula on row $i$ of this vector, and similarly $B_{i}$ for $b$. Function $f$ 's compact representation results in an $n \times n$ matrix, and we use $F_{i j}$ to denote the formula at row $i$, column $j$.

The formula $a . f=b$ is the conjunction of the following $n$ formulas:

$$
\begin{aligned}
\left(A_{1} \wedge F_{11} \vee \cdots\right. & \left.\vee A_{n} \wedge F_{n 1}\right) \Leftrightarrow B_{1} \\
& \wedge \cdots \wedge \\
\left(A_{1} \wedge F_{1 n} \vee \cdots\right. & \left.\vee A_{n} \wedge F_{n n}\right) \Leftrightarrow B_{n}
\end{aligned}
$$

Exactly one of the $A_{i}$ is true, so we can apply Logical Simplification 2:

$$
\begin{aligned}
A_{1} \wedge\left(F_{11} \Leftrightarrow B_{1}\right) & \vee \cdots \vee A_{n} \wedge\left(F_{n 1} \Leftrightarrow B_{1}\right) \\
& \wedge \cdots \wedge \\
A_{1} \wedge\left(F_{1 n} \Leftrightarrow B_{n}\right) & \vee \cdots \vee A_{n} \wedge\left(F_{n n} \Leftrightarrow B_{n}\right)
\end{aligned}
$$

We can then apply Logical Simplification 1: 


$$
\begin{aligned}
\left(\neg A_{1} \vee\left(F_{11} \Leftrightarrow B_{1}\right)\right) & \wedge \cdots \wedge \neg\left(A_{n} \vee\left(F_{n 1} \Leftrightarrow B_{1}\right)\right) \\
& \wedge \cdots \wedge \\
\left(\neg A_{1} \vee\left(F_{1 n} \Leftrightarrow B_{n}\right)\right) & \wedge \cdots \wedge\left(\neg A_{n} \vee\left(F_{n n} \Leftrightarrow B_{n}\right)\right)
\end{aligned}
$$

After moving terms around and factoring, we obtain:

$$
\begin{gathered}
\neg A_{1} \vee\left(\left(F_{11} \Leftrightarrow B_{1}\right) \wedge \cdots \wedge\left(F_{1 n} \Leftrightarrow B_{n}\right)\right) \\
\cdots \\
\neg A_{n} \vee\left(\left(F_{n 1} \Leftrightarrow B_{1}\right) \wedge \cdots \wedge\left(F_{n n} \Leftrightarrow B_{n}\right)\right)
\end{gathered}
$$

Note that for all $i$, the formulas $F_{i 1}, \cdots, F_{i n}$ and $B_{1}, \cdots, B_{n}$ satisfy the conditions of Logical Simplification 3. So we apply it to obtain:

$$
\begin{gathered}
\left(\neg A_{1} \vee\left(f_{11} \Leftrightarrow b_{1}\right) \wedge \cdots \wedge\left(f_{1 l} \Leftrightarrow b_{l}\right)\right) \\
\cdots \\
\left(\neg A_{n} \vee\left(f_{n 1} \Leftrightarrow b_{1}\right) \wedge \cdots \wedge\left(f_{n l} \Leftrightarrow b_{l}\right)\right)
\end{gathered}
$$

Therefore, formula $a . f=b$ results in $2 n l$ clauses, and no additional intermediate variables. The formula $v \cdot f_{1} \cdot \cdots \cdot f_{k_{1}}=u . g_{1} \cdot \cdots . g_{m}$ results in $2 n l k$ clauses, and since we added $k$ variables to break it down, it has $l k$ intermediate boolean variables.

Consider translating $v . f_{1}, \cdots . f_{k_{1}}=u . g_{1}, \cdots . g_{k_{2}}$ to CNF, without using optimizations. Each subexpression of the form a.f results in a vector of $n$ formulas, that are disjunctions of $n$ conjunctions. For each of these formulas, we introduce $n$ propositional variables to rename the conjunctions, requiring 3 clauses each for their definitions. We also introduce 1 variable to rename the whole formula, and its definition requires $n+1$ clauses. So the subexpression a.f requires $n(n+1)$ additional variables, and $n(4 n+1)$ clauses. Therefore there are $n(4 n+1) k$ clauses and $n(n+1) k$ variables after the translation of each side of the equality. The equality itself adds $2 n^{2}$ clauses. We obtain the numbers summarized in the following table.

\begin{tabular}{ccc} 
& Clauses & Intermediate Variables \\
\hline Non-Optimized & $(4 k+2) n^{2}+k n$ & $k n^{2}+k n$ \\
\hline Optimized & $2 k n\lfloor\log (n)\rfloor+k$ & $k\lfloor\log (n)\rfloor+k$ \\
\hline
\end{tabular}

\section{Example}

We illustrate our optimizations on an implementation of insertion in red-black trees [5] (Figure 2]). The code contains two classes RBNode and RBTree, and procedure RBInsert, which performs insertion into a red-black tree. This code is a close transcription of pseudocode presented in a popular algorithms book [5].

We are interested in checking that the red-black invariants are preserved: i.e. that all red nodes must have black children (Inv1), and that all paths leading 

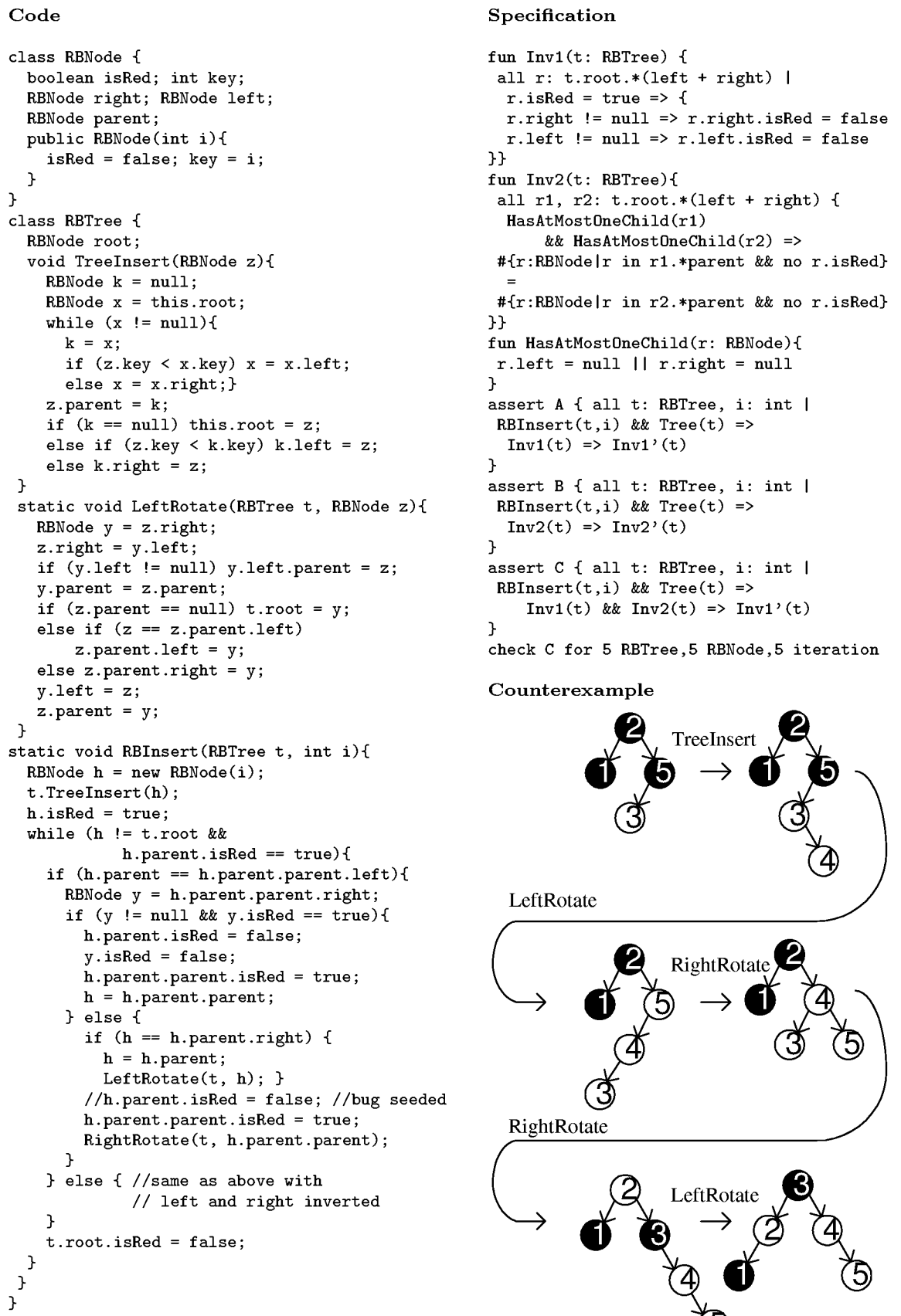

\section{Counterexample}

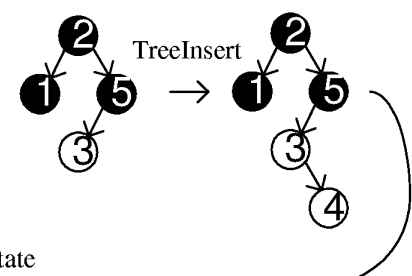

LeftRotate

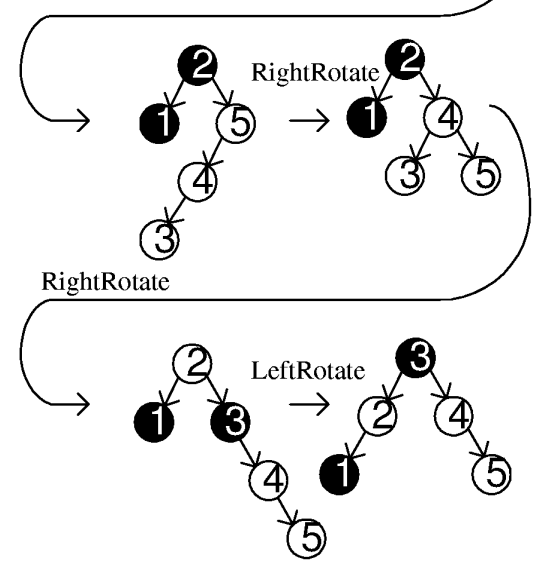

Fig. 2. Insertion into a Red-Black Tree and its Analysis 


\begin{tabular}{|c|c|c|c|c|c|c|c|c|c|}
\hline assertion & scope & \# iter & counter? & time opt & clauses opt & vars opt & time & clauses & vars \\
\hline \multirow[t]{11}{*}{$\mathrm{A}$} & 2 & 2 & no & 0 & 6827 & 2218 & 0 & 15343 & 7638 \\
\hline & 3 & 3 & no & 0 & 14443 & 4201 & 0 & 40042 & 18727 \\
\hline & 4 & 4 & no & 3 & 35642 & 7887 & 20 & 82678 & 36316 \\
\hline & 4 & 10 & no & 4 & 79496 & 15033 & 82 & 188452 & 81148 \\
\hline & 4 & 20 & no & 13 & 152586 & 26943 & - & - & - \\
\hline & 5 & 5 & no & 22 & 58056 & 13384 & 232 & 153259 & 64575 \\
\hline & 5 & 10 & no & 43 & 102281 & 19854 & 506 & 283780 & 117344 \\
\hline & 5 & 20 & no & 25 & 190731 & 32794 & - & - & - \\
\hline & 6 & 6 & no & 159 & 85160 & 19659 & - & - & - \\
\hline & 6 & 10 & no & 198 & 126700 & 25247 & - & - & - \\
\hline & 6 & 20 & no & 514 & 230550 & 39217 & - & - & - \\
\hline \multirow[t]{13}{*}{$\mathrm{B}$} & 2 & 2 & no & 0 & 7066 & 2310 & 0 & 15545 & 7694 \\
\hline & 3 & 3 & no & 0 & 16300 & 4816 & 0 & 41827 & 19281 \\
\hline & 4 & 4 & yes & 7 & 40950 & 9559 & 15 & 87654 & 37766 \\
\hline & 4 & 10 & yes & 25 & 84804 & 16705 & 87 & 193404 & 82574 \\
\hline & 4 & 20 & yes & 109 & 157894 & 28615 & - & - & - \\
\hline & 5 & 5 & yes & 12 & 87369 & 22109 & 52 & 182058 & 72853 \\
\hline & 5 & 10 & yes & 44 & 131594 & 28579 & - & - & - \\
\hline & 5 & 20 & yes & 144 & 220044 & 41519 & - & - & - \\
\hline & 6 & 6 & yes & 22 & 148548 & 38027 & 103 & 318908 & 121400 \\
\hline & 6 & 10 & yes & 49 & 190088 & 43615 & - & - & - \\
\hline & 6 & 20 & yes & 132 & 293938 & 57585 & - & - & - \\
\hline & 7 & 7 & yes & 141 & 240865 & 62127 & - & - & - \\
\hline & 7 & 10 & yes & 189 & 276652 & 66627 & - & - & - \\
\hline \multirow{14}{*}{$\begin{array}{c}\mathrm{C} \\
\text { bug seeded }\end{array}$} & 2 & 2 & no & 0 & 6867 & 2242 & 0 & 15537 & 7726 \\
\hline & 3 & 3 & no & 0 & 15165 & 4471 & 0 & 41172 & 19162 \\
\hline & 4 & 4 & no & 2 & 37886 & 8679 & 18 & 85840 & 37450 \\
\hline & 4 & 10 & no & 12 & 81302 & 15753 & 82 & 192286 & 82630 \\
\hline & 4 & 20 & no & 62 & 153662 & 27543 & - & - & - \\
\hline & 5 & 5 & yes & 18 & 75141 & 18608 & 65 & 172059 & 70344 \\
\hline & 5 & 10 & yes & 43 & 118921 & 25013 & 140 & 303460 & 123573 \\
\hline & 5 & 20 & yes & 100 & 206481 & 37823 & - & - & - \\
\hline & 6 & 6 & yes & 77 & 124936 & 31383 & 202 & 298686 & 116224 \\
\hline & 6 & 10 & yes & 82 & 166056 & 36915 & - & - & - \\
\hline & 7 & 7 & yes & 93 & 199567 & 50690 & - & - & - \\
\hline & 7 & 10 & yes & 239 & 234991 & 55145 & - & - & - \\
\hline & 8 & 8 & yes & 272 & 349823 & 82336 & - & - & - \\
\hline & 8 & 10 & yes & 641 & 386261 & 86216 & - & - & - \\
\hline
\end{tabular}

Fig. 3. Results

to a node with at most one child have the same number of black nodes (Inv2). These invariants maintain a roughly balanced tree.

Figure 2 shows these invariants in Alloy. The function Inv1 says that for all RBNodes $r$ that are reachable from the root of $t$ by following zero or more left or right fields (t.root.*(left+right)), have the property that if $r$ is red, then both its children are black. The second property says that for all RBNodes $r 1$ and r2 reachable from the root, if they both have at most one child2 (indicated by the calls to function HasAtMostOneChild), then the cardinality of the set consisting of all black nodes on the path from $r 1$ to the root is equal to the cardinality of the corresponding set for $\mathrm{r} 2$. The expression \#e denotes the cardinality of e, that is the number of tuples in it.

\footnotetext{
${ }^{2}$ In the original algorithm 5, trees have null leafs that are considered to be black. We do not have these; this is why we need the HasAtMostOneChild function.
} 
The properties are followed by a series of assertions to be checked. For example, assertion A says that for all RBTrees $t$ and all integers $i$, if the procedure RBInsert $(t, i)$ is called and $t$ is a well-formed tree (indicated by Tree $(t)$ ), then the Inv1 property is preserved. A primed version of a function indicates the application of the corresponding property in the post-state, in this case the final state of RBInsert ( $t, i)$, whereas an unprimed version indicates its application the initial state.

We checked these assertions using a prototype implementation for our analysis, which translates Java code directly to CNF using the optimizations, uses Alloy to translate the specifications to CNF, and conjoins the two. The results are shown in Figure 3. Times are in seconds. All experiments were run on a $1.1 \mathrm{GHz}$ PentiumIII with $640 \mathrm{MB}$ of memory, using the BerkMin SAT solver 8]. Some experiments were done after injecting a bug by removing one line in the code (indicated in Figure 2 by the comment bug seeded). In Figure 3] dashes indicate either that the experiment took more than 10 minutes or that there was a shortage of memory. The non-optimized experiments are done by translating a subset of Java code to Alloy and uses the Alloy Analyzer equipped with BerkMin as well.

Some of these experiments result in a counterexample. For instance, the counterexample corresponding to assertion $\mathrm{C}$ with a bug seeded, for scope of 5 and 5 iterations, is shown in Figure 2, The numbers on each node indicate the keys, and red nodes are shown in white. The counterexamples goes through 5 iterations to violate assertion 3 .

The results show that the translation without optimization can obtain all the counterexamples very rapidly. This was expected; a fundamental assumption of our work, which we refer to as the small scope hypothesis, is that most bugs can be demonstrated with small counterexamples. The results also show that the analysis scales better with the optimizations. An empirical study [1] demonstrates that a scope of 6 is enough to obtain full statement and branch coverage for a variety of benchmarks. Our optimizations allow checking all assertions with a scope of 6 , and as high as scope of 8 in some cases.

We can also increase the number of iterations to 20 and get an outcome within a minute or two in most cases. For RBInsert, 20 iterations for each loop correspond to 1540 lines of code. A state-of-the-art SAT solver can handle formulas with up to about 300,000 clauses. These results suggest that a code fragment of 1500 lines might be encodable within these limits. Of course, the tractability of the subsequent analysis is another matter. And in this case, since it is clear that fewer iterations suffice, the solver timings should be taken with a grain of salt.

\section{Related Work}

For a bounded instance of a program, our analysis explores all the possible inputs and executions, typically accounting for billions of cases. Unlike testing, it can also produce an initial configuration of the heap which leads to a property 
violation. It differs from finite state verification tools, such as model checking, in that it is modular: procedures may be checked in isolation without requiring a driver. It differs from shape analysis in that it produces sound counterexamples and no false alarms. It also requires no intermediate code annotations, or userprovided abstractions.

Finite State Verification. FeaVer [10] is a verification tool for C source code, based on the model checker Spin 9]. It extracts a model of a program automatically using a look-up table of abstractions provided by the user. The model is then verified with Spin, which outputs a counterexample when a property is violated. FeaVer has been used successfully to uncover hundreds of bugs in Lucent's PathStar call processing system.

Bandera [4] is a tool that allows analyzing Java source code with different verification tools. It extracts a finite state model of code using slicing and userprovided data abstraction. The result of the extraction is a model that may be mapped into several model checkers (SMV, Spin) and theorem provers (PVS). Unlike Feaver and Bandera, our analysis does not support user-provided abstraction.

The Java PathFinder [17] is an environment for checking Java bytecode, that integrates model checking, program analysis, and testing. It allows userprovided abstractions of the program, and uses the Bandera tool for slicing. Java PathFinder requires an initialization of the heap that fixes it to a particular configuration. Thus it is impossible to have the tool automatically find an initial configuration that breaks an assertion, as it can be done in our analysis.

The SLAM [2] tool is designed to check if a program obeys API usage rules. It does not require user annotations, and is fully automatic. It abstracts a program into a boolean program that is a conservative approximation. The boolean program is then subjected to reachability analysis to see if an error state is attainable. If this is not the case, then there is a guarantee that the original program cannot reach the error state. If an error state is reachable, then it is analyzed automatically to see if it is part of a feasible execution, in which case a counterexample is output to the user. If no feasible execution leads to the error state, then appropriate predicates are added to the abstraction, again automatically. The process then starts over with this refined abstract program. SLAM targets temporal safety properties, and not structural properties.

Shape Analysis. Shape analysis algorithms [16] can identify invariants for programs that manipulate heap-allocated storage. They represent the heap as shape graphs, conservative abstractions that capture properties at different points in the program. The parametric shape analysis (PSA) [16] method uses a 3-valued logic to represent shape graphs, and is a framework that can be instantiated with different instrumentation predicates that retain more refined information about concrete heaps, and can help to identify different classes of properties. Unlike our method, PSA can prove properties without bounds, but it may issue false alarms. In contrast to our property-independent translation, PSA requires 
instrumentation predicates that tailor the analysis for the discovery of particular properties. Recent work [14] presents space-efficient encodings of boolean formulas that represent shape graphs. Its goals not its methods are similar to ours.

Theorem Proving. The Extended Static Checker [6] uses a specialized theorem prover to check code against user-specified specifications. Structural properties such as those handled in our analysis are not expressible. Experience has shown that ESC requires many intermediate code annotations, making it less practical. An extension of ESC, the VeriFun tool [7, uses predicate abstraction, decision procedures, and automated successive refinement. It requires no user annotation beyond the property to be checked. It differs with our analysis in that it cannot readily handle the kinds of structural properties we consider here.

\section{Conclusions}

We presented a suite of optimizations that results in much improved scalability for our analysis of object-oriented code, which targets structural properties of the heap, requires no user-annotation, and outputs no false alarms. Our optimizations are a suite of simple but judiciously chosen logical simplifications, that are not incremental, i.e. their composition results in an improvement in scalability.

A conventional way to scale an analysis such as this is to require user-provided specifications for all procedures. The ability to handle longer code sequences allows a longer procedure to be considered, and for smaller procedures, it allows specifications to be omitted. The scalability of this analysis is therefore crucial to allow checking code fragments in which specifications are written at a coarser, more economical, granularity.

Our experimental results use a prototype tool that translates a subset of Java directly to CNF using the optimizations, while the non-optimized tool translated to Alloy, and therefore benefitted from the Alloy Analyzer's internal simplifications. As part of future work, we plan to incorporate our optimizations in the Alloy Analyzer, so that we can benefit from its simplifications as well. We also plan to run more experiments on different code bases, to further investigate the effect of our optimizations.

Acknowledgements. This work has greatly benefited from discussions with Manu Sridharan and Ilya Shlyakhter, and comments from anonymous reviewers. It was funded in part by ITR grant \#0086154 from the National Science Foundation, by a grant from NASA, and by an endowment from Doug and Pat Ross. The first author thanks Joan Wheelis for her unbounded support, and dedicates this paper to the memory of her grandfather, Reza Safavi Golpayegani. 


\section{References}

1. A. Andoni, D. Daniliuc, S. Khurshid, and D. Marinov. "Evaluating the Small Scope Hypothesis", MIT Laboratory for Computer Science, September 2002. Unpublished manuscript.

2. T. Ball, S. K. Rajamani. "The SLAM Project: Debugging System Software via Static Analysis", Proc. POPL 2002, January 2002.

3. D. R. Chase, M. Wegman and F. Zadeck. "Analysis of Pointers and Structures", Proc. Conf. on Programming Language Design and Implementation, 1990.

4. J. C. Corbett, M. B. Dwyer, J. Hatcliff, S. Laubach, C. S. Pasareanu, Robby, H. Zheng. "Bandera: Extracting Finite-State Models from Java Source Code", Proc. International Conference on Software Engineering, June 2000.

5. T. H. Cormen, C. E. Leiserson, R. L. Rivest. "Introduction to Algorithms", MIT Press, 1990.

6. D. Detlefs, K. R. Leino, G. Nelson, and J. Saxe. "Extended Static Checking". Technical Report 159, Compaq Systems Research Center, 1998.

7. Cormac Flanagan. Personal communication.

8. E. Goldberg and Y. Novikov. "BerkMin: A fast and robust SAT-solver", In Design, Automation, and Test in Europe, March 2002.

9. G.J. Holzmann. "The Model Checker Spin", IEEE Trans. on Software Engineering, Vol. 23, 5, May 1997.

10. G. J. Holzmann and M. H. Smith. "Automating Software Feature Verification", Bell Labs Technical Journal, Vol. 5, 2, April-June 2000.

11. Daniel Jackson. "Automating First-Order Relational Logic", Proc. ACM SIGSOFT Conf. Foundations of Software Engineering, San Diego, November 2000.

12. D. Jackson, I. Shlyakhter and M. Sridharan. "A Micromodularity Mechanism", Proc. ACM SIGSOFT Conf. Foundations of Software Engineering, 2001.

13. D. Jackson and M. Vaziri. "Finding Bugs with a Constraint Solver", Proc. International Conference on Software Testing and Analysis, August 2000.

14. R. Manevich, G. Ramalingam, J. Field, D. Goyal, M. Sagiv. "Compactly Representing First-Order Structures for Static Analysis", In Proc. SAS 2002, 2002.

15. D. A. Plaisted and S. Greenbaum. "A Structure-Preserving Clause Form Translation", Journal of Symbolic Computation, 2:293-304, 1986.

16. M. Sagiv, T. Reps, and R. Wilhelm. "Parametric shape analysis via 3 -valued logic", In ACM Transactions on Programming Languages and Systems, 24(3), 217-298, 2002.

17. W. Visser, K. Havelund, G. Brat and S. Park. "Model Checking Programs", International Conference on Automated Software Engineering, September 2000. 